\title{
Um Método Quantitativo de Avaliação para Filtragem da Interferência Frente-Verso em Documentos Coloridos
}

\author{
João Marcelo Monte da Silva, Rafael Dueire Lins e Ismael Gomes Netto
}

\begin{abstract}
Resumo- Sempre que se tem um documento escrito em ambos os lados de papel translúcido diz-se que há uma interferência frente-verso. A literatura técnica descreve vários algoritmos para filtragem da interferência frente-verso em documentos. Este artigo apresenta um novo método quantitativo para avaliar tais algoritmos. Este método é baseado na síntese de imagens com tal interferência e em uma posterior comparação entre as imagens filtradas e uma imagem de referência fazendo uso do PSNR.
\end{abstract}

Palavras-Chave-Análise de Algoritmos, Interferência frenteverso, Documentos Históricos.

Abstract - Whenever a document is written on both sides of translucent paper there is a back-to-front interference, also known as bleeding or show-through. In the literature there are many algorithms to filter out the back-to-front interference in documents. This paper presents a new quantitative method to assess those algorithms. This method is based on the synthesis of images with interference.

Keywords- Algorithm Assessment, Back-to-front interference, Bleeding, Show-through, Historical Documents.

\section{INTRODUÇÃO}

Quando um documento é escrito ou impresso em ambos os lados de um papel cuja opacidade permite que a impressão do verso seja vista na frente há uma degradação da legibilidade desse documento, dificultando sua transcrição e binarização. Esse fenômeno, primeiramente citado na literatura técnica por Lins em 1994 [1], recebeu o nome de "interferência frente-verso" (do inglês back-tofront interference), mais adiante, outros autores também o chamaram de bleeding e show-through. Em documentos históricos há vários casos em que a tinta, devido a sua alta fluidez, permeia o papel, intensificando ainda mais a visibilidade do conteúdo impresso do verso na face frontal do documento. A carta de Joaquim Nabuco mostrada na Figura 1, pertencente ao acervo da Fundação Joaquim Nabuco - FUNDAJ [2], exemplifica tal interferência.

$\mathrm{Na}$ literatura há vários algoritmos que foram projetados para filtrar a interferência frente-verso [3], [4], [5], [6], [7], [8], [9]. Este artigo descreve um método quantitativo para a avaliação de tais algoritmos quando aplicados em imagens de documentos coloridos.

João Marcelo Monte da Silva, Rafael Dueire Lins e Ismael Gomes Netto, Departamento de Eletrônica e Sistemas, Centro de Tecnologia e Geociências, Universidade Federal de Pernambuco, Recife, Brasil, E-mails: joaommsilva@gmail.com,rdl@ufpe.br, ismaelgomesn@gmail.com.

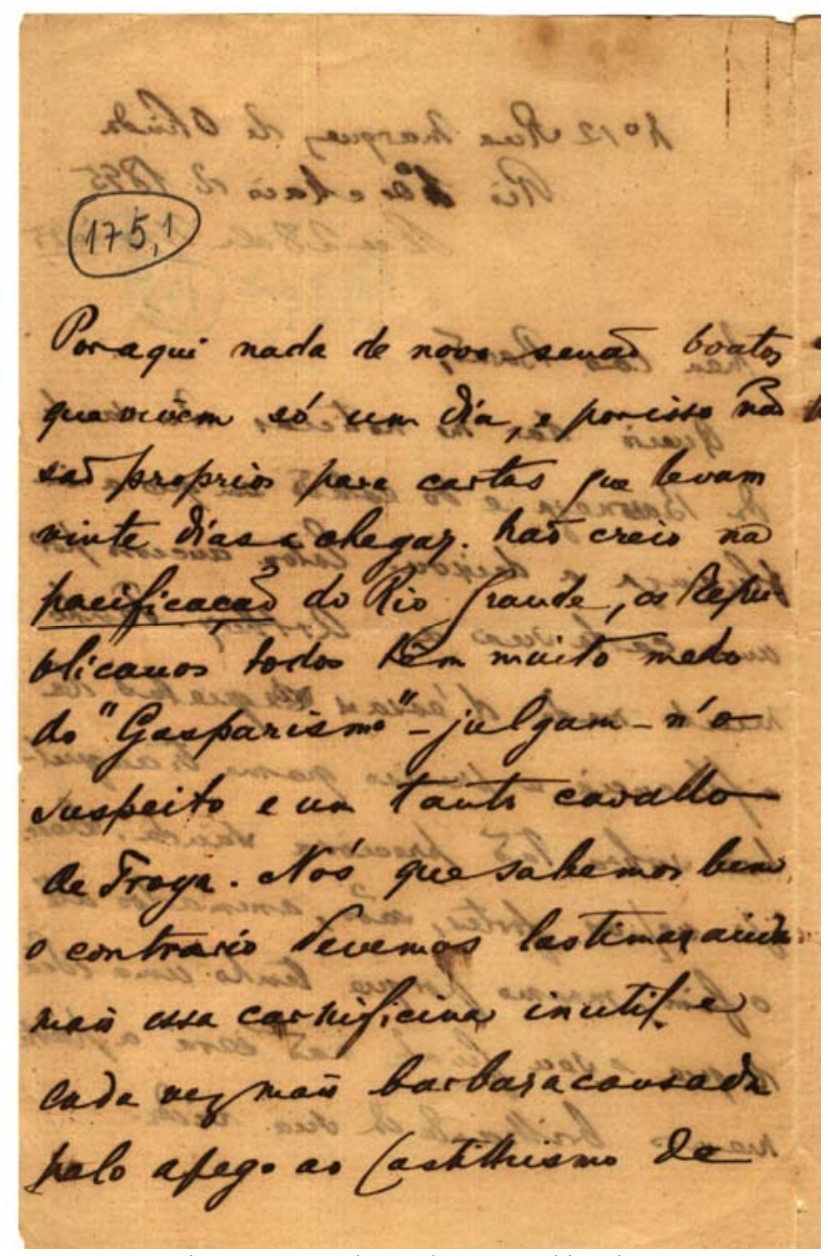

Fig. 1. Imagem de um documento histórico com interferência frente-verso.

O processo aqui desenvolvido é uma "generalização" do apresentado em [10] que lida com imagens em níveis de cinza. A partir do método aqui descrito, é possível comparar os algoritmos de filtragem de uma forma justa.

Para demonstrar o método quantitativo que será descrito na seção II, far-se-á uso de três algoritmos. O primeiro é um algoritmo que será introduzido neste trabalho, este faz uso do conceito de filtragem em espelho [1] e será descrito na seção III. Os outros dois são algoritmos de filtragem por limiar, sendo um com seleção manual de pontos [3] e o outro automático [4]. Os resultados e as análises serão discutidos na seção IV. Finalmente, na seção V, conclusões e algumas linhas para trabalhos futuros. 


\section{Descrição do MÉtodo de AVALIAÇÃo QUANTITATIVO}

O método aqui descrito é dividido em dois passos:

1. síntese de imagens com interferência frente-verso baseado em duas imagens sem interferência; e

2. comparação das imagens filtradas através dos algoritmos com uma imagem de referência - fazendo uso do PSNR (Peak Signal to Noise Ratio) para cada componente de cor no espaço RGB.

O PSNR foi escolhido por ter uma baixa complexidade computacional frente a parâmetros que definem a qualidade da imagem no sentido perceptual [11].

\section{A. Síntese de Imagens com Interferência Frente-Verso}

Esta etapa tem início tomando-se duas imagens de documentos coloridos sem interferência frente-verso, como mostra a Figura 2: F - representa a frente do documento; e $\mathrm{V}-\mathrm{o}$ verso do mesmo.

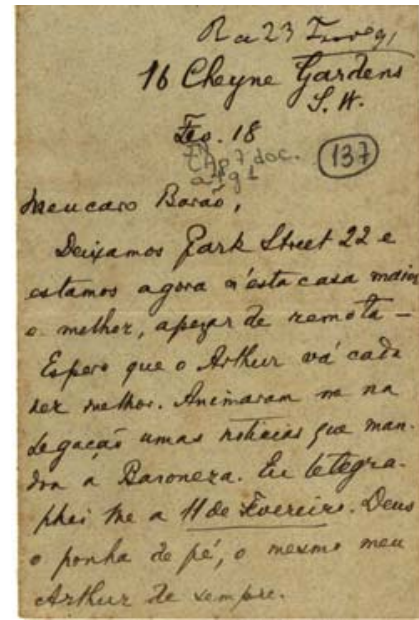

Imagem Frente - F

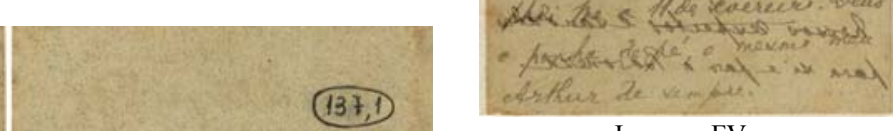

Imagem $\mathrm{FV}_{\alpha}$

Fig. 3. Composição, usando canal alfa das imagens $\mathrm{F}$ e $\mathrm{V}$.

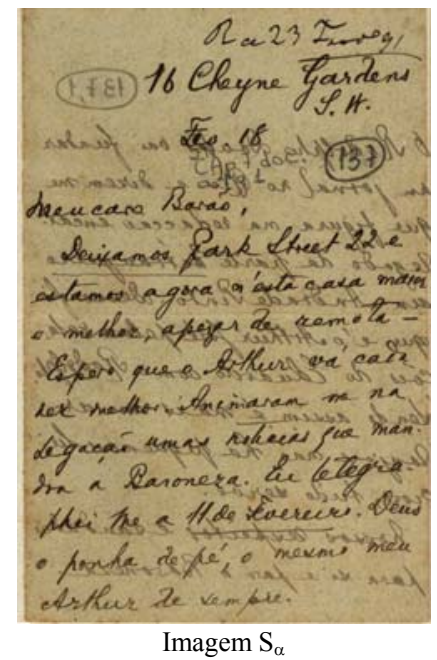

Fig. 4. Resultado final da síntese.
- $\quad$ Sejam $f^{(\mathrm{Y})}(i, j)$ e $f v_{\alpha}^{(\mathrm{Y})}(i, j)$ os valores de luminância dos pixels das imagens $\mathrm{F}$ e $\mathrm{FV}_{\alpha}$, respectivamente, na posição $(i, j)$ determinados por:

$\left\{\begin{array}{l}f^{(\mathrm{Y})}(i, j)=0.299 f^{(\mathrm{R})}(i, j)+0.587 f^{(\mathrm{G})}(i, j)+0.114 f^{(\mathrm{B})}(i, j) \\ f v_{\alpha}^{(\mathrm{Y})}(i, j)=0.299 f v_{\alpha}^{(\mathrm{R})}(i, j)+0.587 f v_{\alpha}^{(\mathrm{G})}(i, j)+0.114 f v_{\alpha}^{(\mathrm{B})}(i, j)\end{array}\right.$

onde $\left(f^{\mathrm{R})}(i, j), f^{(\mathrm{G})}(i, j), f^{\mathrm{B})}(i, j)\right)$ e $\left(f v_{\alpha}^{(\mathrm{R})}(i, j), f v_{\alpha}{ }^{(\mathrm{G})}(i, j)\right.$, $\left.f v_{\alpha}{ }^{(\mathrm{B})}(i, j)\right)$ são as componentes (vermelho,verde,azul) dos pixels das imagens $\mathrm{F}$ e $\mathrm{FV}_{\alpha}$, respectivamente, na posição $(i, j)$.

- $\quad$ Se $f^{(\mathrm{Y})}(i, j) \leq f v_{\alpha}^{(\mathrm{Y})}(i, j)$, ou seja, se o pixel proveniente de $\mathrm{F}$ tiver uma luminância mais escura ou igual a do pixel vindo de $\mathrm{FV}_{\alpha}$, então o pixel da imagem final $\mathrm{S}_{\alpha}$ terá a cor do pixel de F. Caso contrario, ele terá a cor do pixel proveniente de $\mathrm{FV}_{\alpha}$. Isto é mostrado na Equação 3 . por:

$$
\left\{\begin{array}{l}
\mathrm{FV}_{\alpha}^{(\mathrm{R})}=\alpha \mathrm{F}^{(\mathrm{R})}+(1-\alpha) \mathrm{V}^{(\mathrm{R})} \\
\mathrm{FV}_{\alpha}^{(\mathrm{G})}=\alpha \mathrm{F}^{(\mathrm{G})}+(1-\alpha) \mathrm{V}^{(\mathrm{G})} . \\
\mathrm{FV}_{\alpha}^{(\mathrm{B})}=\alpha \mathrm{F}^{(\mathrm{B})}+(1-\alpha) \mathrm{V}^{(\mathrm{B})}
\end{array}\right.
$$

onde os sobrescritos (R), (G) e (B) denotam as componentes de vermelho, verde e azul das imagens $\mathrm{FV}_{\alpha}, \mathrm{F}$ e V, e $\alpha$ é o coeficiente de opacidade da imagem $\mathrm{F}$ (frente) que varia de 0 a $100 \%$. Ainda, o fator $(1-\alpha)$ indica o coeficiente de transparência da imagem $\mathrm{F}$.

Para se ter uma melhor visualização do que está ocorrendo, imagine a imagem $\mathrm{F}$ como um vidro colorido. A cor de cada ponto é a cor do vidro que tem opacidade $\alpha$ mais a cor que está atrás reduzida pelo fator $(1-\alpha)$ que representa a transparência do vidro.

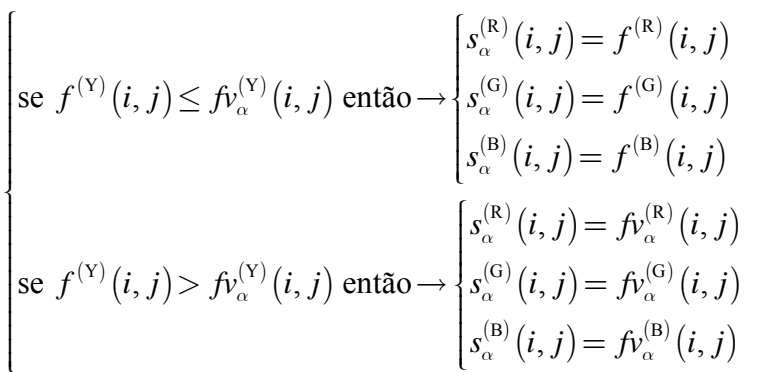

onde $s^{(\mathrm{R})}{ }_{\alpha}(i, j), s^{(\mathrm{G})}{ }_{\alpha}(i, j)$ e $s^{(\mathrm{B})}{ }_{\alpha}(i, j)$ são as componentes vermelho, verde e azul, respectivamente, do pixel da imagem $\mathrm{S}_{\alpha}$ na posição $(i, j)$.

$\mathrm{Na}$ Figura 5 pode ser visto um diagrama de bloco do processo de síntese. A imagem indicada por $\mathrm{V}$ é a imagem $\mathrm{V}$ da Figura 2 espelhada. 


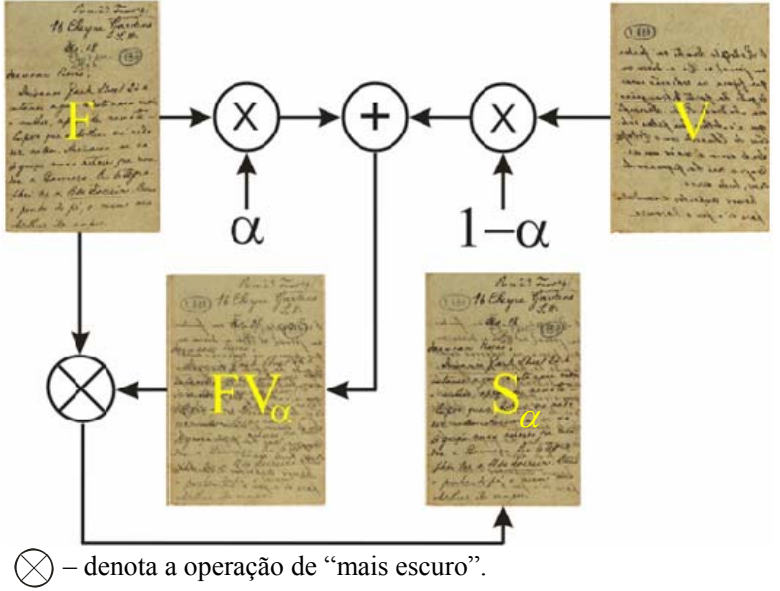

Fig. 5. Diagrama de bloco da etapa de síntese.

Para avaliar a capacidade de filtragem dos algoritmos variamos o fator de opacidade $\alpha$ de 0 a $100 \%$ $(\alpha=0,1, \ldots, 100 \%)$. O efeito da variação de $\alpha$ na imagem final $\mathrm{S}_{\alpha}$ gerada pelos documentos mostrados na Figura 2 com V espelhada é apresentado na Figura 6.
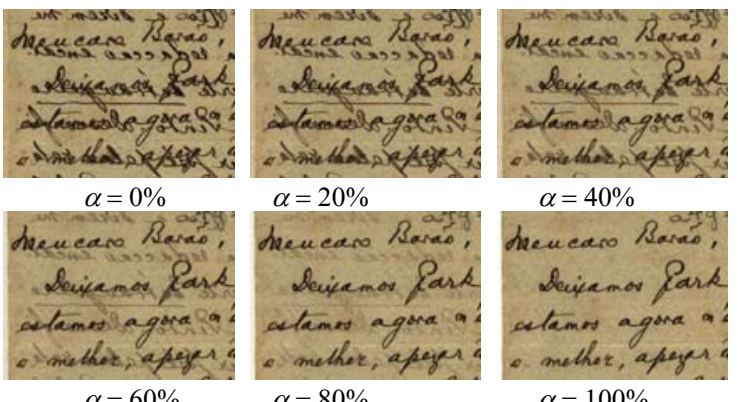

Fig. 6. Partes de imagens sintetizadas $\mathrm{S}_{\alpha}$ com diferentes valores de $\alpha$.

\section{B. Cálculo dos PSNR's}

Após o processo de síntese, as imagens sintetizadas são filtradas pelos algoritmos a serem avaliados. Os resultados das filtragens são comparados com a imagem F (vide Figura 2). O PSNR (Peak Signal-to-Noise Ratio) será usado nesta etapa para medir a qualidade das imagens resultantes do processo de filtragem. Como se está trabalhando com imagens em true-color o PSNR será computado para cada componente de cor RGB como segue

$$
\operatorname{PSNR}_{k}^{(c o r)}(\alpha)=20 \log _{10} \frac{255}{\sqrt{\frac{\sum_{i=1}^{A} \sum_{j=1}^{L}\left[f^{(c o r)}(i, j)-f i l t_{\alpha, k}^{(c o r)}(i, j)\right]^{2}}{A \times L}}},
$$

onde $A$ é a altura da imagem, $L$ é a largura da imagem, $f^{(c o r)}(i, j)$ é a intensidade da componente cor na posição $(i, j)$ da imagem F (referência) e filt ${ }_{\alpha, k}{ }^{(c o r)}(i, j)$ é a intensidade da componente cor na posição $(i, j)$ da imagem FILT $_{\alpha, k}$ que é o resultado da filtragem da imagem $\mathrm{S}_{\alpha}$ pelo algoritmo $k$.

A seguir, na Figura 7, é apresentado um diagrama de bloco que ilustra o processo de comparação entre a imagem de referência $\mathrm{F}$ e a filtrada FILT $_{\alpha, k}$.

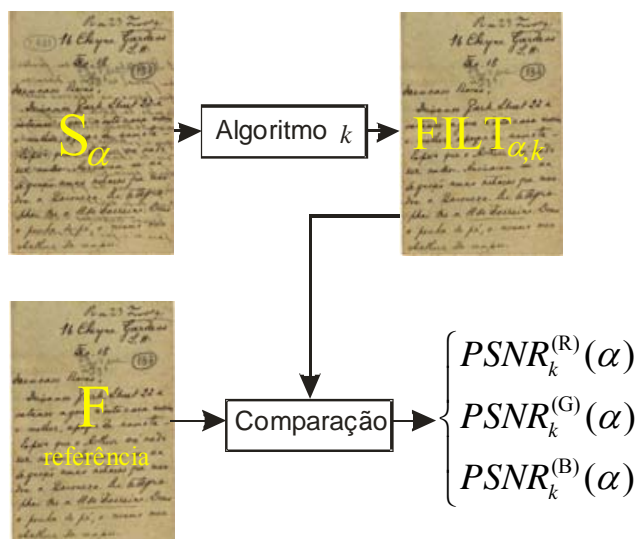

Fig. 7. Diagrama de bloco que ilustra o processo de comparação entre a imagem de referência $\mathrm{F}$ e a filtrada $\mathrm{FILT}_{\alpha, k}$.

\section{AlgoritMo BASEADO EM FILTRAGEM EM ESPELHO}

Salienta-se que o algoritmo aqui descrito foi concebido apenas para a demonstração do método.

Este algoritmo além de usar a imagem a ser filtrada, ele necessita da imagem do verso do documento para inferir quais pixels pertencem à interferência. Dessa forma, só é indicada sua utilização quando se têm as imagens da frente e do verso do documento a ser filtrado.

$\mathrm{O}$ algoritmo funciona da seguinte forma:

1. tomam-se as duas faces de um documento, a frente e o verso refletido (vide Figuras $8 \mathrm{a}$ e $8 \mathrm{~b}$ );

2. alinham-se, o máximo possível, as duas imagens de forma que a interferência constante na imagem da frente fique na mesma posição que o texto da imagem do verso refletido;

3. comparam-se, pixel a pixel, as imagens em níveis de cinza da frente e do verso refletido, destacando-se como interferência os pixels cujos níveis de cinza são mais baixos na segunda do que na primeira, ou seja, se um dado pixel da imagem do verso refletido é mais escuro do que seu correspondente na imagem da frente ele é classificado como interferência (Vide Figura 8c);

4. preenche-se a área destacada como interferência com pixels cujas cores são próximas da moda do documento (vide Figura 8d).

Deve-se salientar que o segundo passo acima descrito não é uma tarefa trivial, pois um detalhe pode fazer com que as imagens nunca tenham um bom alinhamento, por exemplo, dobraduras e amassados no documento original quando digitalizadas impossibilitam o perfeito alinhamento das duas imagens.

O preenchimento da área destacada (quarto passo do algoritmo) é feito da seguinte forma:

i. toma-se uma grande área do documento, tendo o cuidado de não incluir sua borda;

ii. observa-se, na área tomada, a luminância mais freqüente $\left(y_{\text {moda }}\right)$, a luminância de maior valor $\left(y_{\max }\right)$ e a diferença entre elas $d=y_{\max }-y_{\text {moda }}$ (vide Figura 9);

iii. calcula-se o valor do $\operatorname{limiar} T=y_{\text {moda }}-d$;

iv. varre-se a área definida armazenando-se em um vetor - amostra_do_papel - os pixels (RGB) que tiverem 
um valor de luminância - nível de cinza - maior que $T$;

v. preenche-se, finalmente, a área interferente escolhendo-se aleatoriamente pixels do vetor amostra_do_papel.

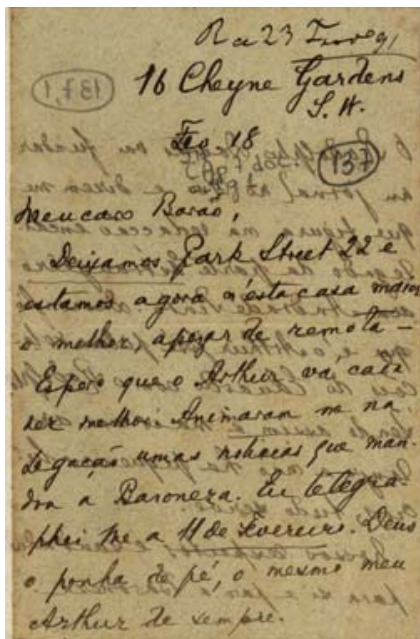

(a)

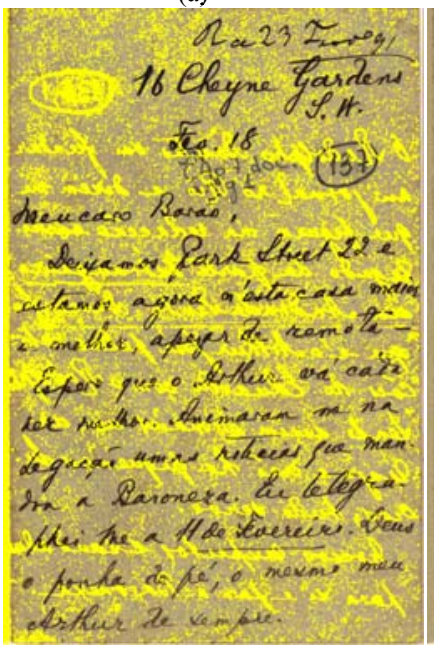

(c)

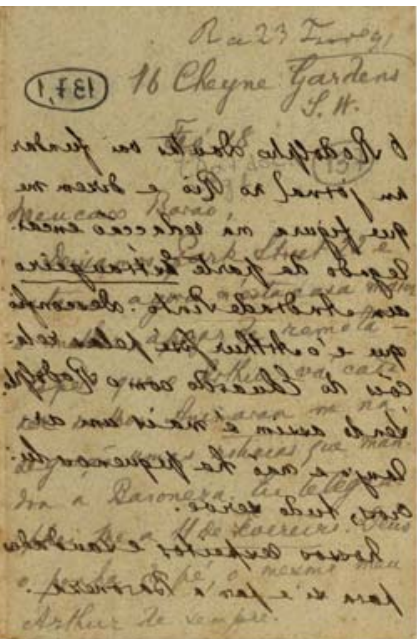

(b)

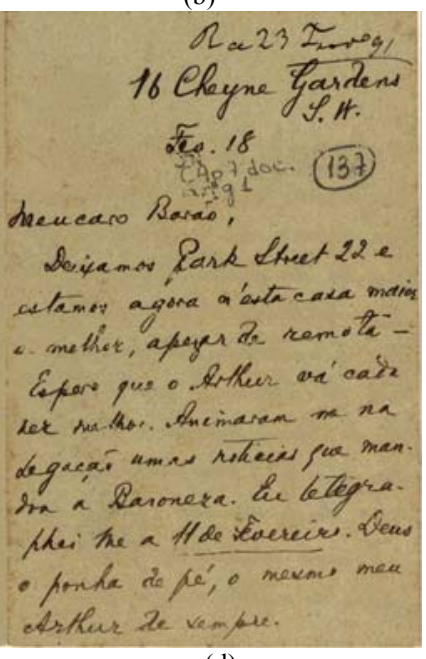

(d)
Fig. 8. (a) Face da frente e (b) do verso refletida de um documento sintetizado com interferência frente-verso. (c) Imagem da Figura 8a com os pixels da interferência destacados de amarelo e (d) resultado final da filtragem.

O passo iii. acima descrito é justificado pelo fato da distribuição dos níveis de cinza do papel terem uma certa simetria em relação a sua moda (vide Figura 9).

A forma de filtragem em espelho foi primeiramente sugerida por Lins [1]. Este conceito foi utilizado nos algoritmos propostos por Sharma [5] e por Cao, Tan e Shen [6].

As imagens mostradas na Figura 8 são imagens sintetizadas pelo processo descrito na seção anterior. Tais imagens foram escolhidas para a demonstração deste algoritmo por estarem "naturalmente" alinhadas, devido ao processo de síntese utilizado, pois como já mencionado o alinhamento não é uma tarefa trivial.

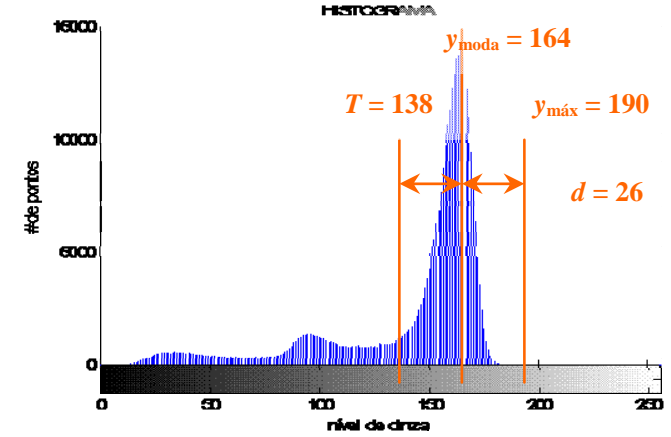

Fig. 9. Histograma da janela referente à versão em níveis de cinza da imagem da Figura 8a.

\section{ANÁLISES E RESULTADOS}

Foram escolhidos três algoritmos para demonstração do método de avaliação quantitativo descrito na seção II.

- Algoritmo 1: filtragem em espelho - faz uso da imagem do verso do documento para classificar os pixels da interferência (este algoritmo está descrito na seção III);

- Algoritmo 2: filtragem por limiar manual [3] baseado na seleção manual de alguns pixels da imagem original para encontrar a interferência;

- Algoritmo 3: filtragem por limiar automático [4] utiliza um algoritmo de limiarização para demarcar a área interferente.

Como mencionado na descrição do método foram sintetizadas 101 imagens com intensidades diversas de interferência frente-verso $(\alpha=0,1, \ldots, 100 \%)$. Essas imagens foram filtradas pelos algoritmos aqui mencionados. Em seguida, foram calculados os PSNR's para cada componente de cor de cada imagem, como indicado na seção II, e seus resultados podem ser vistos nos gráficos da Figura 10. Tais gráficos trazem no eixo das abscissas o valor da opacidade $\alpha$ utilizado para sintetizar a imagem $\mathrm{S}_{\alpha}$ correspondente, e no eixo das ordenadas os valores dos PSNR's das componentes de cor RGB.

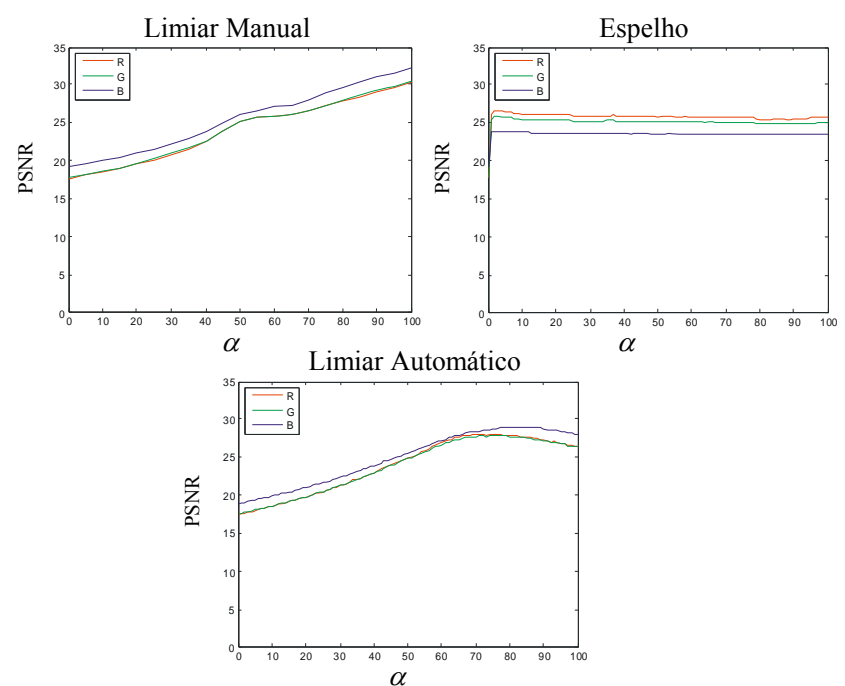

Fig. 10. Gráficos dos PSNR's das imagens filtradas FILT $_{\alpha, k}$ em relação à imagem $\mathrm{F}$ 
É possível observar através dos gráficos apresentados na Figura 10 que o algoritmo de filtragem em espelho é o mais estável, e, em linhas gerais, tem o melhor desempenho. Este método é bastante eficiente na separação da interferência do resto do documento. Contudo, além do algoritmo necessitar da imagem do verso do documento para fazer a discriminação dos pixels interferentes, há a dificuldade no alinhamento das imagens, uma tarefa não trivial. Seu alto desempenho é justificado pelo fato das imagens já estarem alinhadas devido à forma com que foi realizado o processo de síntese.

O segundo colocado foi o algoritmo de filtragem por limiar manual. Este por selecionar manualmente alguns pixels (tinta da frente, interferência e papel) do documento faz uma melhor discriminação dos pixels interferentes do que o método de filtragem por limiar automático. Contudo, o último tem a vantagem de não fazer uso da imagem do verso do documento nem de precisar da intervenção do usuário na escolha de pixels do mesmo.

Observa-se, ainda na Figura 10, que o algoritmo de filtragem por limiar manual aumenta seu desempenho quando a interferência vai se tornando mais fraca, ou seja, quando o valor de $\alpha$ cresce. $\mathrm{O}$ mesmo não ocorre com o algoritmo de filtragem em espelho, pois mesmo em imagens com baixa interferência este algoritmo classifica como interferência os pixels que são mais escuros na imagem do verso. Dessa forma, sendo a interferência quase inexistente, tal algoritmo classifica pixels que pertencem ao papel, como interferência. Para melhorar seu desempenho quanto a este fato, pode-se utilizar a diferença $\delta$ entre os pixels da frente e do verso, e dispor de um limiar $T_{\delta}$ para determinar se o pixel pertence ou não à interferência, em outras palavras, se a diferença $\delta$ for positiva e menor do que $T_{\delta}\left(0<\delta<T_{\delta}\right)$ o pixel em questão será classificado como interferência, caso contrário não será classificado como tal. Assim, em imagens com interferência muito fraca, o algoritmo não classificará como interferência pixels que pertencem ao papel, pois a diferença calculada provavelmente será maior que o limiar estabelecido.

Deve-se destacar que o algoritmo de filtragem por limiar automático tem um comportamento semelhante ao algoritmo de filtragem em espelho em relação à classificação dos pixels interferentes para interferência fraca $(\alpha>80)$. Sendo a interferência quase inexistente o algoritmo classifica como interferência pixels do papel. Entretanto ele tem uma tendência semelhante a do manual para valores de $\alpha<80$.

Do conjunto de imagens sintetizadas foram selecionadas três amostras. As amostras escolhidas foram tais que representassem interferências existentes em documentos reais do acervo de documentos digitalizados de Joaquim Nabuco [2]. As amostras com $\alpha=40 \%, \alpha=65 \%$ e $\alpha=90 \%$ (vide Figura 11) representam imagens com interferências forte, média e fraca, respectivamente.

Na Figura 12 são apresentadas as imagens da Figura 11 filtradas pelos três algoritmos aqui comparados.

Todo este estudo tem a finalidade de indicar qual o algoritmo mais apropriado para atuar em uma determinada faixa de valores de $\alpha$. Dessa forma, é interessante saber determinar o valor de $\alpha$ em um dado documento.

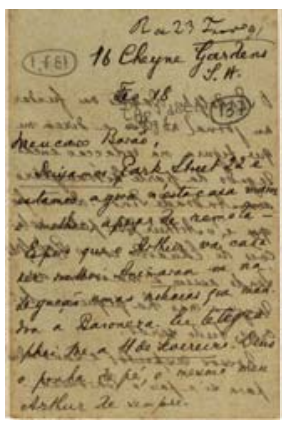

(a) $\alpha=40 \%$

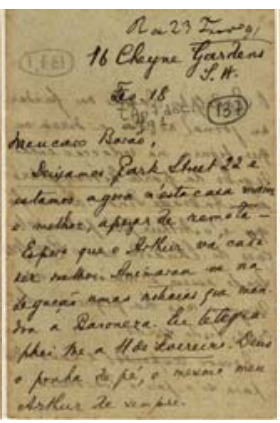

(b) $\alpha=65 \%$

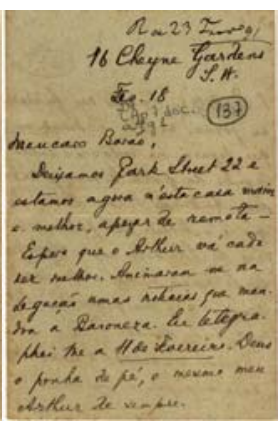

(c) $\alpha=90 \%$
Fig. 11. Imagens sintetizadas (a) $\alpha=40 \%$, (b) $\alpha=65 \%$ e (c) $\alpha=90 \%$.

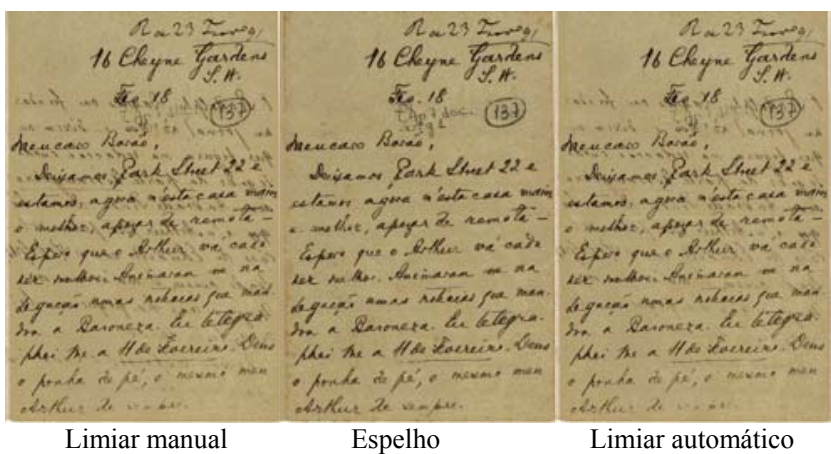

(a) $\alpha=40 \%$

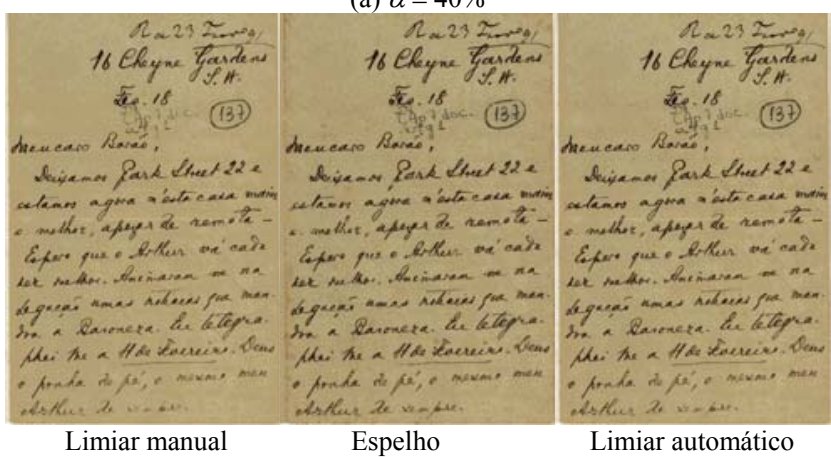

(a) $\alpha=65 \%$

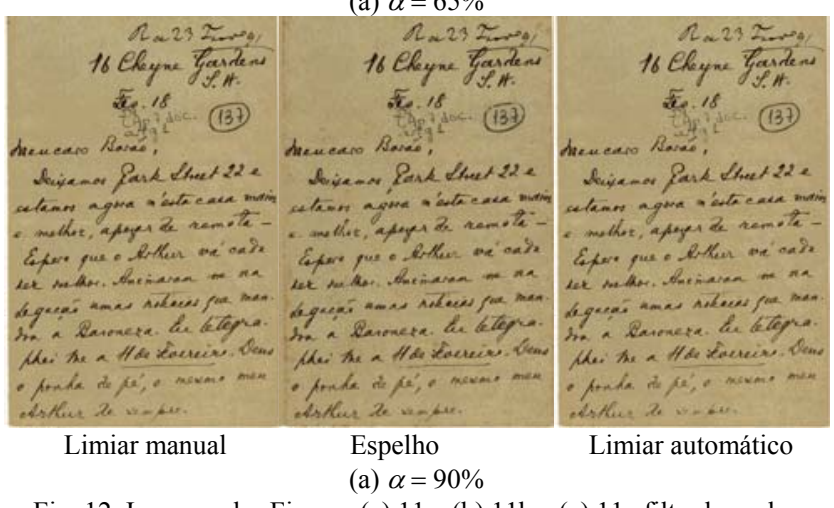

Fig. 12. Imagens das Figuras (a) 11a, (b) $11 \mathrm{~b}$ e (c) 11c filtradas pelos algoritmos comparados.

È possível estimar a opacidade $\alpha$ de um documento real da seguinte forma:

- pelo modelo apresentado, um pixel de interferência tem intensidade luminosa interf $(i, j)$ dado por:

$\operatorname{interf}(i, j)=\alpha \cdot \operatorname{papel}(i, j)+(1-\alpha) \cdot$ tinta $_{\text {verso }}(i, j)$

onde papel $(i, j)$ e tinta $a_{\text {verso }}(i, j)$ são as intensidades dos pixels do papel e da tinta do verso, respectivamente; 
- na grande maioria dos casos pode-se assumir que a intensidade da tinta do verso tinta $a_{\text {verso é }}$ aproximadamente a mesma da tinta da frente tinta $a_{\text {frente }}$, dessa forma, pode-se coletar amostras da interferência, do papel e da tinta da frente para representarem as intensidades interf, papel e tinta $_{\text {frente, }}$, respectivamente;

- por fim, usando a Equação 5 e assumindo que tinta $a_{\text {verso }} \approx t i n t a_{\text {frente, }}$, podemos estimar o valor de $\alpha$ por:

$$
\alpha=\frac{\text { interf }- \text { tinta }_{\text {frente }}}{\text { papel }- \text { tinta }_{\text {frente }}}
$$

Para exemplificar, toma-se a imagem da Figura 1. Os valores coletados foram:

$$
\left\{\begin{array}{l}
\text { tinta }_{\text {frente }}=23 \\
\text { interf }=106 \\
\text { papel }=201
\end{array} \Rightarrow \alpha=\frac{106-23}{201-23} \approx 0,47\right.
$$

assim, pode-se dizer que tal imagem tem um coeficiente de opacidade $\alpha \approx 47 \%$.

Uma maneira automática de se saber qual o valor da interferência pode ser obtida através da binarização direta da imagem colorida. A experiência mostra que cerca de no máximo $8 \%$ dos pixels de um documento são traduzidos em pixels pretos. A binarização direta implicará em uma quantidade maior de pixels, proporcional ao coeficiente de opacidade. O relacionamento das faixas com a quantidade de pixels ainda não está estabelecida, ficando para trabalhos futuros.

\section{CONClusões E Trabalhos Futuros}

Foi introduzido neste trabalho um método quantitativo para a avaliação de algoritmos que filtram a interferência frente-verso em imagens de documentos coloridos. Esse método permite a realização de um estudo sobre tais algoritmos com a finalidade de saber qual o algoritmo mais indicado para filtrar um determinado documento, tomando como base a opacidade $\alpha$. Para se automatizar essa escolha é necessário se estimar, aproximadamente, valores das amostras da tinta da frente, do papel, e da tinta interferente para então se calcular a opacidade $\alpha$. Outra forma é binarizar diretamente a imagem colorida, e através de uma relação pré-estabelecida determinar a opacidade $\alpha$ através da quantidade de pixels pretos da imagem binarizada. Com o valor da opacidade determinado, escolhe-se o melhor algoritmo para a filtragem, tomando como base um estudo previamente feito.

O PSNR foi utilizado para medir a qualidade da imagem final. Em trabalhos futuros, pretende-se definir medidas que informe a legibilidade da informação útil, a preservação do papel e a qualidade do preenchimento da área interferente de forma independente.

Em muitas imagens a interferência aparece "borrada". Para se levar em conta esse efeito em um estudo, deve-se passar a imagem que irá representar o verso por um filtro passa baixa antes do processo de síntese. O modelo pode ser ainda mais sofisticado, fazendo uso de um filtro adaptativo que leva em conta dados da imagem da frente.

\section{AGRADECIMENTOS}

Ao CNPq - Conselho Nacional de Pesquisas e Desenvolvimento Tecnológico, Governo do Brasil - pelo suporte financeiro e à FUNDAJ - Fundação Joaquim Nabuco pela permissão de utilização das imagens.

\section{REFERÊNCIAS}

[1] R. D. Lins, et al. "An Environment for Processing Images of Historical Documents. Microprocessing \& Microprogramming”, pp. 111-121, North-Holland, 1994.

[2] FUNDAJ: www.fundaj.gov.br

[3] R. D. Lins e I. G. Netto. "Um Ambiente para Filtrar a Interferência Frente-Verso em Documentos Históricos”. SBrT-2007, Recife, Brasil, 2007.

[4] J. M. M. da Silva e R. D. Lins. "Um Novo Método de Filtragem de Interferência Frente-Verso em Documentos Coloridos”. SBrT-2007, Recife, Brasil, 2007.

[5] G.Sharma, "Show-through cancellation in scans of duplex printed documents", IEEE Trans. Image Processing, v10(5):736-754, 2001.

[6] R. Cao, C.L.Tan and P.Shen, "A wavelet approach to double-sided document image pair processing", Proc. Int. Conf. Image Proc. Oct. 2001.

[7] Feng Su and Ali Mohammad-Djafari, "Bayesian Separation of Document Images with Hidden Markov Model", 2nd International Conference on Computer Vision Theory and Applications, Barcelona, Spain, 2007.

[8] B. Ophir and D. Malah, "Improved cross-talk cancellation in scanned images by adaptive decorrelation", 23rd IEEE Convention of Electrical and Electronics Engineers in Israel, 2004, pp. 388-391.

[9] H.Nishida and T.Suzuki, "A Multiscale Approach to Restoring Scanned Color Document Images with Show-trough Effects", Proc. of. ICDAR 2003, 2003.

[10] R. D. Lins and J. M. M. da Silva. "Assessing Algorithms to Remove Back-to-Front Interference in Documents", ITS-2006, Fortaleza, Brazil, 2006.

[11] R. F. Zampolo e R. Seara. "Estudo Comparativo entre Métricas para Avaliação da Qualidade de Imagens". XXII SIMPÓSIO BRASILEIRO DE TELECOMUNICAÇÕES, pp. 237-241, Campinas-SP, 2005. 